\title{
IL MISTICISMO FEMMINILE
}

\author{
WOMEN'S MYSTICISM
}

Gualtiero De Santi

Universidad de Urbino, Italia

\section{Riassunto}

Si racconta la relazione intima di alcune figure femminili, famose o ignote, con Dio; relazione completamente diversa da quella degli uomini con Dio. Le donne in Umbria a partire dal XIII secolo andavano acquistando libertà religiosa e ciò si traduce nella produzione di testi spesso sottovalutati che raccontano di esperienze mistiche dotate di forza straordinaria e tutt'affatto personali. Il rapporto con Dio si fa a volte drammatico e conflittuale. Queste donne mistiche, colte o analfabete che fossero, si fecero promotrici di movimenti religiosi.

\section{Parole chiave:}

Dio, donne, visione, incontro, anima.

\section{Abstract:}

It tells about the intimate relationship of some well-known or unknown women with God. The relationship is completely different from the men's one. Women in Umbria started to conquer religious freedon starting from the 13th century. This can be noticed from several texts that are often underestimated that tell us about mystical experiences with an extra ordinary power and very personal. The relationship can become dramatic and adversarial. These women, sometimes educated and other times unlitterate, promoted many religious movements.

\section{KeY WORD:}

God, women, vision, encounter, soul. 
Il giorno della purificazione della beata vergine, quando il mattino nella chiesa dei frati minori di Foligno si distribuiscono le candele, mi venne fatto un discorso che diceva: "Questa è l'ora in cui la madonna giunse col figlio al tempio". Quando l'anima udì ciò, provò un amore così grande che nulla di esso può esser detto o capito. E subito l'anima fu elevata, e vide la madonna entrare proprio in quell'ora; allora l'anima le andò incontro con grande timore e reverenza. La madonna assicurò l'anima; poi stese verso di me il suo figliolo e disse: "Prendilo, innamorata del figlio mio". Così dicendo, stese le braccia e depose nelle mie il suo figliolo; questi aveva gli occhi chiusi come se dormisse, ed era stretto nelle bende, tutto fasciato. La madonna, come stanca del viaggio, si sedette. Aveva atteggiamenti e gesti così graziosi e soavi che era una cosa bellissima il guardarla; l'anima guardava non solo il bambino che tenevo in braccio, ma anche la madonna. A un tratto mi trovai fra le braccia il bambino tutto nudo. Apri gli occhi, li alzò verso di me, cominciò a guardarmi. Nell'esser così guardata, trovai un tale amore che mi sopraffece. Accostai il mio volto al suo, la mia guancia toccò la sua; ne seguì, all'aprirsi degli occhi di colui che mi trovai così nudo fra le braccia, una gioia indicibile; non ne posso dir nulla (Da Foligno, 1992: 219-220).

La citazione è acclusa alle cosiddette Istruzioni di Angela da Foligno, brevi frammenti inseriti nella seconda parte del suo Liber, congiuntamente a varie esortazioni e missive. Come anche nel caso dell'opera maggiore, appunto il Libro dell'Esperienza, i brani non sono di pugno di Angela, la quale era illetterata e dialettofona e che dunque non sapeva né scrivere né leggere, ma bensì di religiosi che le stavano dietro in quel suo inesausto girovagare per le campagne e i centri dell'Umbria, nel tentativo di trasferire in scrittura quanto lei andava profferendo e altrettanto per mitigarne e addolcirne gli ardori. Lei gridava in volgare e loro volgevano in latino.

Nondimeno, la lingua del delirio mistico non avrebbe potuto agevolmente modularsi nella lingua della moderazione e dell'obbedienza maschile. La trasposizione in altro stato mentale e emotivo - quanto interviene nel caso di Angela da Foligno e dei suoi trascrittori - si può pensare in una forma di idioma a interfaccia, che nel caso in questione va rigorosamente affrontato e valutato. Non c'è dubbio che esso emargini gli eccessi ma esso non impedisce il venire alla luce di quella parte femminile che ricondurremmo alla maternità. Sotto il linguaggio ufficiale, scivola insomma la manifestazione di un continuum materno che già prelude alla scoperta della relazione personale con il divino.

L'ibridazione o mescidazione che ne risulta non sfugge all'interrogativo se questa scrittura riesca ad esprimere il legame con Dio. Anche nei casi al limite, data la sempre eccessiva impetuosità, torna a proporsi il problema di una peculiarità femminile: una differenza che, storicamente in quel declinare del Duecento, teneva in non cale nelle sue manifestazioni l'ortodossia religiosa. La verità era quella naturale e non invece quella ufficiale. Ciò perché la fede in Dio era sentita come spazio interiore raccolto nella dimensione singola, sia soprattutto per la totale conformità di quella ortodossia al pensiero e al potere maschile.

In un libro relativamente recente che ha potuto godere anche di una versione spagnola, Il Dio delle donne di Luisa Muraro (che ha costituito il nostro sottotesto critico insieme alla lettera fattane da Erminia Macola), l'autrice assegna proprio alla tensione mistica il compito di sostenerla nel penetrare più a fondo nella dimensione femminile. Sceglie per ciò stesso di attraversare le immagini del divino appartenute alle donne secondo la testimonianza offerta dai libri che ci restano. La domanda posta è se il Dio che la percezione femminile ha incontrato o ha creduto di scorgere nelle proprie visioni e nei propri trasalimenti, un Dio con il quale le mistiche vantano una confidenza e una prossimità ignote agli uomini, riesca in qualche modo a entrare in circolo col presente. Se sia utile insomma per indiziare i caratteri di quella differenza che segue altri cammini.

L'impegno della saggista è ovviamente pari allo sforzo di ripercorrere l'esperienza viva di alcune figure famose o ignote che la storia religiosa ci ha consegnato (o che abbiamo riscoperto): meglio, il loro stare nella relazione con l'Altro quando l'Altro si chiami Dio, in quell'ininterrotto movimento e scambio che si viene a creare tra amante e amato. In termini teologici l'atto che si compie è parlare con Gesù e farlo esprimere in una lingua materna - viva, naturale, spontanea - che si pensa appannaggio del femminile.

Quando questo è potuto accadere - nel corso del XIII secolo in Umbria ma altrettanto nel centro Europa sempre in relazione alla temperie religiosa emersa in quegli anni - si è venuta espandendo una libertà sino allora inimmaginabile e mai prima conquistata dalle donne. Una libertà di ordine religioso, beninteso: ma nella piena autonomia di una spiritualità tenuta al riparo dal controllo maschile pur se in procinto di rientrare nei primi decenni del Trecento sotto l'egida della Chiesa ufficiale.

Il filtro che guida le ricerche e l'interpretazione è infine l'esperienza delle donne catturate dalla passione religiosa, quale ci è stata tramandata in testi il più delle volte ignorati e sottovalutati, quando non propriamente confinati e reclusi in archivi e sotto la polvere di biblioteche vescovili e conventuali. Testi in gran parte controversi e ancor oggi da interrogare e da decrittare (ad es. Lo specchio delle anime semplici che condusse Margherita Porete sul rogo il 1 giugno 1310), in altra parte considerati esercizi mistici al femminile, deprivati di razionalità e saggezza teologica.

Osserva Luisa Muraro:

Del movimento religioso delle donne nel Medioevo - cui dobbiamo un numero notevole di testi e alcuni capolavori - non esiste una narrazione storica unitaria, 
come esiste invece delle lotte per il potere politico-religioso o delle rivolte sociali. Non si può nemmeno dire esattamente come quel movimento sia cominciato e quando sia finito. Meglio cosi: possiamo pensare che non sia mai finito. Di esso abbiamo molte tracce e alcuni documenti la cui energia di pensiero, a distanza di tanti secoli, non è spenta. Si trattò di una lotta che possiamo considerare politica; ma in questione non era il potere politico né la giustizia sociale. la lotta era per un senso più grande epú libero del nostro stare al mondo. Per la felicità, sì, credo che questa sia la parola giusta. Il terreno di lotta era l'ordine simbolico, intendo l'ordine invisibile ma attivo e operante - lo impariamo imparando a parlare - cui attiene il rapporto tra quello che c'è e quello che non c'è, o non si vede, tra le cose e le parole, tra i corpi e i segni, tra i desideri e la legge, tra l'esperienza e la possibilità di dire il vero (Muraro, 2003: 26).

Si tratta di ripercorrere e insieme ricomprendere nelle più profonde implicazioni i vissuti e lo spirito di quelle figure femminili (la appena menzionata Porete, poi Hadewijch d'Anversa, Mechthild von Magdeburg, ovviamentela “Lella” daFoligno) che hanno anticipato esperienze più recenti (Thérèse de Lisieux). Il riscontro storicamente accertato e determinabile di gruppi e movimenti che sull'onda della febbre religiosa dell'XII-XIII secolo riuscirono a sfuggire al mantello maschile e ecclesiastico - basterà solo rammemorare il fenomeno del Beghinaggio, che si manifestò fra il XII e il XIV sec. nelle Fiandre e nel nord della Francia, ma altrettanto in Belgio e in Germania - va a supportare l'ipotesi di caratteristiche intrinseche al femminile proprio nell'atto di condursi alla volta del divino.

Il predicato essenziale ancorché problematico, se non proprio controvertibile, è che quando si volge alla cosa anelata la donna cessa di recludersi in un ordine predeterminato - sociale, teologico, patriarcale - muovendosi verso un'alterità di gran lunga più radicale e netta di quanto mai intervenga in un uomo. È, per addurre un esempio, ciò che Simone Weil ha definito astensione dal dogma nell'atto del rapporto con Dio:

Les dogmes de la foi ne sont pas des choses à affirmer. Ce sont des choses à regarder à une certaine distance, avec attention, respect et amour. C'est le serpent d'airain dont la vertu est telle que quiconque le regarde vivra. Ce renard attentif et aimant, par un choc en retour, fait jaillir dans l'âme une source de lumière qui illumine tous les aspects de la vie humaine ici-bas

dès qu'on les affirme (Weil, 1951: 50).

In questo rapporto che si apre tra la donna e Dio, pur data evidentemente la grande disparità intercorrente tra i due, scivola una intimità singolare, e una franchezza incondizionata. Vedi Santa Caterina che ragiona di Cristo alla stregua di un innamorato; ed ecco le beghine, e non solo loro, che si pongono con pieno agio e disponibilità nella posizione femminile accettando la differenza, dunque accettando la forza e l'energia dell'altro. Purché l'altro sia però Dio, cioè senza limiti.
Insomma, quando e se nel loro rapporto col divino le donne appunto incontrano il loro Dio, cioè la relazione, sospendono ogni aspetto "rigido, strutturante, sintattico, ordinatore di Dio, che organizza visioni fisse delle cose e ordini che vanno conservati, riaffermati, imposti" (Macola, 2003: 13). Il Dio delle donne - osserva ancora Muraro - è straripante e non va confuso con quello degli uomini il quale è invece trascendente. Allorquando un fiume esce dagli argini non si limita ad oltrepassarli, li rompe.

Potrebbe esser questa la differenza tra San Francesco d'Assisi e la Beata Angela da Foligno (lui che si effonde in un Cantico che lega creato e creature, divino e mondo, vita e morte; lei che si affretta trafelata per tutta l'Umbria e quando fa il suo ingresso all'interno della Basilica di Assisi tanto è colma di emozioni e scossa che la debbono allontanare con la forza per il clamore che solleva).

Ed è la differenza - almeno credo - tra un Juan de la Cruz e Teresa de Jesús. Il santo poeta per dono di estaticità appare recluso in una interiore distillazione spirituale. Lei all'incontrario è pronta a trarre gioie dal corpo e dalla visione dell'amato, a godere della sua presenza. Colpisce che nel Libro de la vida ricorra tanto frequentemente il verbo gozar, che ha il valore di assaporamento vorace, carnale. (E il Monte do gozo è quello dei pellegrini della Via Lattea quando al vertice di una piccola erta scorgono finalmente ai loro piedi la cittadina di Santiago de Compostela). Più contenuta e precisa nella determinazione teologica, Caterina Benincasa scrive a Frate Giovanni di Bindo di Doccio de' Frati di Monte Oliveto: “Oh quanto è beata l'anima che corre e consuma la vita sua in vera e santa virtù! Perocché in questa vita gusta l'arra di vita eterna" (S. Caterina da Siena, 1939: II, 18)

L'altra scena che vede insieme congiuntamente Dio e la donna, si annuncia però nei modi di una forza dirompente, che investe accesamente il corpo. Qualcosa sopravviene all'improvviso: travolgente e affatto distruttivo. Qualcosa che conquista e dà godimento ma pur mette a dura prova. Le fitte e gli spasimi, gli affanni dell'animo, infine i sintomi fisici sono da leggere quali altrettante verifiche dell'incontro. La Santa Teresa del Bernini è l'esempio di come quella forza tanto imperiosa si presenti, e quale possa esserne l'efficacia sull'abito umano. Tutto è allora - dopo l'incontro, dopo l'esperienza - sofferenza e gaudio, trascinamento dei sensi e dolore.

Ma è il punto in cui irrompe la domanda al Signore: che significato ha tanta violenza amorosa e cosa egli in fatto cerca? A quel punto Dio si personalizza e comincia per davvero ad esistere. Da entità metafisica si trasforma in un particolare soggetto. Ed allora - come si riscontra nell'autobiografia teresiana - da elemento caratterizzante di un sistema teoretico e culturale, diventa l'evento decisivo di una pratica. Dio è lo stimolo che guida energicamente verso una dimensione spirituale per niente discinta di ali teologiche. Per questo abbiamo una Chiara d'Assisi, ben attenta a muoversi nel complicato universo della Chiesa con le sue colte e determinate lettere in latino; e persino 
l'analfabeta Angela sa raggiungere vette teologiche (cosi da essere dottrinalmente menzionata da Teresa de Ávila e persino dichiarata "magistra theologorum"). E anche nel caso delle beghine si è arrivati all'esplicitazione di una teologia in una lingua materna, cioè femminile.

E tuttavia quel cerchio che s'avvita dal passato pretende di inanellarsi al presente. Ecco allora - quasi a riprodurre uno di quei fatti misteriosi che avvenivano in passato, in anni di passione palingenetica - la testimonianza della mistica e laica Simone Weil centrata su un avvenimento occorso a lei alle falde di Assisi nel 1937, nel momento in cui entrava nella piccola cappella romanica del XII sec. in Santa Maria degli Angeli. È giusto in quellattimo che un impulso potente obbliga alla genuflessione proprio lei, che mai sino a quel momento si era inginocchiata in una chiesa: "quelque chose de plus fort que moi m'a obligée, pour la première fois de ma vie, à me mettre à genoux" (Weil, 2009: 23). Successivamente, nel corso di una lettura a voce alta di versi di poeti metafisici inglesi del Seicento, l'episodio si chiarisce nel suo lato amoroso e di conquista: "le Christ lui-même est descendu et m’a prise" (Weil, 2009: 25). L'esperienza mistica è insomma coinvolgente e tutt'affatto personale.

Tanta apertura si spinge sino a termini inauditi, impensabili negli uomini. Anche per questo nell'orizzonte delle mistiche medievali (e poi dei secoli posteriori), l'esperienza descritta trascende vistosamente ogni limite. Si resta colpiti dalla delirante irruenza con cui la madre Giovanna degli Angeli fa la storia della possessione diabolica delle Orsoline in quel di Loudun (siamo nel ‘600) e quando si accusa di libertinaggi di mente e di congiungimenti carnali con i demoni. Ancor più si rimane attoniti di fronte a tanti avvenimenti dell'esistenza terrena di Angela da Foligno.

Sintomatico l'episodio dell'abbraccio del Cristo morto, raccontato tramite quel fratel Anselmo che la seguiva passo passo e che era il suo confidente e scrivano:

Il sabato santo che seguì le cose narrate quella fedele cristiana mi raccontò le meravigliose gioie ricevute da Dio. Tra l'altro disse a me, frate scrittore, che in quel giorno lei fedele cristiana, attirata in un eccesso di mente, stette nel sepolcro insieme con Cristo. E disse di aver dapprima baciato il petto di Cristo. Lo vedeva disteso con gli occhi chiusi, come giacque morto. Poi gli baciò la bocca. Diceva che su quella bocca colse un mirabile, inenarrabile, dilettoso odore, che da lì spirava. Disse che ci fu una breve pausa. Disse che poi posò la sua guancia sopra la guancia di Cristo. Allora Cristo posò la sua mano sopra l'altra sua guancia e la strinse a sé. Allora quella fedele cristiana udì, rivolte a sé, queste parole: "Prima che giacessi nel sepolcro ti tenni così stretta a me" E benché realizzasse che Cristo pronunciava quelle parole tuttavia lo vedeva disteso con gli occhi chiusi, con le labbra immobili. Lei stava abbandonata in una gioia inenarrabile (Da Foligno, 1992: 147).

Il rapporto con Dio non è facile, né tantomeno idilliaco. A volte si fa impaurente e drammatico, conflittuale e oscuro: e allora il diavolo può essere confuso con Cristo, le visioni e i vaneggiamenti giungono persino a travolgere. Forse anche per questo, monache e mistiche si ritrovarono sottoposte al rigore dei controlli ecclesiastici nella Spagna del 1500-'600, censurate e in alcuni non rari casi tradotte in carcere. I diari e le confessioni scritte vennero per ciò stesso purgate, rielaborate e riformulate da mano maschile. La scrittura, negata alla base per l'assenza di formazione culturale (tale il caso di Angela), tornava ad essere negata anche quando il livello di preparazione era più che elevato (come nell'esempio di Teresa de Ávila). Il "misticismo intellettuale" di Juana Inés de la Cruz ebbe dura vita in Messico, ma avrebbe incontrato un destino più periglioso in Europa.

Non si deve comunque credere che il Dio visto e anelato dalle donne non possieda una dimensione storica. O meglio, che non abbia a che fare col periodo storico nel quale quelle donne erano vissute. I flussi mistici dei quali parliamo si svilupparono soprattutto nel tardo Medioevo, allora che in parte si andò allentando il controllo della Chiesa. E - in Italia - si configura con tensione e dinamiche particolari in quel dell'Umbria.

In questa regione del centro Italia si creò nel secolo XIII un movimento di mulieres religiosae che in parte andarono a chiudersi all'interno di conventi tuttavia dotandosi $\mathrm{di}$ una loro regola, in parte si liberarono del peso di famiglia e figli per riunirsi in gruppi che girovagavano proclamando e ostentando il loro senso della fede. Tale libertà avrebbe avuto breve durata, ben presto riassorbita e normalizzata dalla Chiesa e dalla società. Ma per la prima volta le donne cercavano Dio senza la mediazione maschile (della chiesa, della società, della famiglia). E grazie a questo - fatto altrettanto straordinario si attribuirono, anzi si conquistarono, il potere della parola e conseguentemente della scrittura.

Nello spazio particolare dell'incontro con il divino, imponevano una dimensione e relazione individuali condividendo con l'Altro quell'amore ideale che le sospingeva verso una mistica sponsale. Angela vede Dio (o san Francesco) nelle visioni che il sogno origina in lei. Nel suo convento di Rupertsberg, nei dintorni di Bingen, la beata Hildegarde è assiduamente sottoposta a una raffica di visioni. Simile in questa alla badessa che l'aveva educata, Jutta von Spanheim, era tuttavia bloccata da un riserbo che le impediva di dare notizia di quanto le accadeva. Poi, però (siamo all'anno di grazia 1141), Dio stesso si premura di ordinarle che mettesse su carta quanto vedeva e sentiva: ma non a suo modo, seguendo il proprio arbitrio, ma invece secondo la volontà di Colui che tutto sa e dispone nelle recondite profondità della mente. Quel mistero e quello spazio interiore che le donne hanno saputo penetrare negli incontri col Creatore.

Le visioni - parliamo ancora di Hildegarde - si ripeterono con frequenza sempre maggiore. A un certo punto iniziarono a trascendere le comuni misure del tempo 
anticipando con diverse rivelazioni il futuro. Per la badessa di Rupertsberg, costituirono quasi una seconda vita. Sempre più la beata trovava difficile riportare e traslitterare quel che le era fatto vedere. Confusa e per niente adusa a parlare, non conosceva la linea da seguire nel trascrivere le pulsioni che riceveva. Sin quando il Signore le chiese espressamente di farlo non alla maniera degli uomini ma al modo in cui avvertiva dentro sé i messaggi che Egli le mandava (da donna amante che riceva quei doni esattamente dal proprio sposo: nei modi dunque della lingua muliebre)

"O quam mirabilis est inspiratio / que hominem sic suscitauit", meravigliosa ispirazione che così ha fatto nascere l'uomo - proclama estatica la badessa (Bingen, 2007: 376). E certo il contenuto di quella evenienza, di pari passo alle visioni delle sante e mistiche del tempo, possiede contorni tutti particolari, nei quali si traduce un subconscio collettivo, cioè il sostrato femminile dell'anima dell'umanità del tempo. L'itinerario psichico illuminato dalla fede avverte la realtà fisica della spiritualità. La contemplazione allucinata produce squarci che non appartengono alla misura del maschile.

Ma sino a un certo punto. Giacché ad esempio, quella "mistica a tavolino" di teologi e pensatori che Hans Urs von Balthasar contrapponeva alla "mistica in ginocchio" di santi e visionari, non è sempre unicamente femminile. Alla stessa stregua di Teresa de Ávila, un san Juan de la Cruz lasciò ampissimo spazio nella sua ascesa verso la divinità al soggettivismo derivante dalle proprie esperienze. E a conferma si legge nella Lettre che Simone Weil ebbe a indirizzare a un religioso: "En fait, les mystiques de presque toutes les traditions religieuses se rejoignent presque jusqu'à l'identité. Ils constituent la vérité de chacune" (Weil, 1951: 49). Tutti, uomini inclusi.

Nondimeno quei bagliori di visionarietà non osservavano unicamente l'indicibile, che le parole delle mistiche si provarono comunque a compitare: ecco il Liber Lelle di Angela, il Miroir des simples âmes anéanties della Porete oppure il poema Das fliessende Licht der Gottheit di Mechtild von Magdeburg. La stessa Hildegarde, madre superiora poetessa e teologa, scrisse e musicò 77 inni di lode a Dio, redasse un Trittico Visionario - Scivas, Liber Vitae Meritorum. Liber Divinorum Operum - in una forma che compendiava tutt'insieme storia sacra e cosmologia. Eppure i suoi testi ebbero anche il compito di ammonire papi e imperatori a che desistessero dalle guerre e dai conflitti che insanguinavano l'epoca. "Ella era una hjia fiel de la Iglesia, pero sus visiones la hicieron el oráculo de espantosas acusaciones" (Fülöp-Miller, 1949: 8). Entrambi, il Papa e l'Imperatore, portavano la responsabilità delle rovine e dei disastri del tempo.

Hildegarde von Bingen fu anche assertrice dell'autonomia religiosa e civile dei conventi nei quali fu madre superiora. Anche in questo, si ritrova una particolare similarità con tante esperienze vissute a migliaia di chilometri dall'Assia renana. Le donne potevano scegliere di stare all'interno dei conventi oppure al di fuori, ma vollero essere loro stesse a darsi le regole. La Beata Giovanna da Orvieto veste all'età di 20 anni l'abito domenicano, ma intende vivere nell'ordine al di fuori della clausura. Chiara d'Assisi contro le decisioni della Chiesa fonda l'Ordine delle Clarisse che si appaiava a quello dei Frati minori francescani (esortando in quattro celebri lettere Agnese di Boemia a fare la stessa cosa seguendo coraggiosamente la propria strada).

Certamente non fu questione di ribellione, ma invece di una fedeltà al proprio universo interiore bonificato e visitato da Dio. Né si trattò di concedersi regole meno austere, allora che è vero il contrario: giacché in genere, furono le donne stesse a produrre una loro severissima disciplina. Nell'Umbria dugentesca Chiara da Montefalco viene eletta badessa del convento di S. Croce dopo aver scelto di isolarsi in un reclusorio sin da quando era bambina. In Spagna Teresa de Ávila rifonda l'ordine del Carmelo.

Come si sa, l'idea di un nuovo monastero le nasce sul finire del 1560. Scrive nel Libro de la Vida: “Pensaba qué podría hacer por Dios, y pensé que lo primero era seguir el llamamiento que Su Majestad me había hecho a religión, guardando mi Regla con la mayor perfección que pudiese" (De Jesús, 1994: 222).

La realizzazione del progetto venne ordinata da una speciale grazia mistica (la visione dell'Inferno e il desiderio di liberare le anime) ma anche dal colloquio che ella ha con alcune amiche. “Ofrecióse una vez, estando con una persona, decirme a mí y a otras, que si no seríamos para ser monjas de la manera de las descalzas, que aun posible era poder hacer un monasterio" (De Jesús, 1994: 222). L'ipotesi è che un numero esiguo di donne, undici o dodici, si dedichi alla vita di orazione e alle pratiche della mortificazione e dell'isolamento, secondo le norme originarie dettate dai padri del Carmelo. Le opposizioni e i dissensi fioccarono numerosi, in primo luogo da parte delle consorelle del Monastero dell'Incarnazione, poi da parte della città. Finalmente il Breve apostolico di autorizzazione consentì di avviare la progettata riforma. Così il 24 agosto del 1562 Teresa fondò ad Ávila il primo monastero intitolato a San Giuseppe. Un secondo Carmelo sarebbe stato innalzato a Medina del Campo cinque anni dopo.

Ma più singolare che in quella stessa annata 1567 Teresa avesse in incontro con Juan de la Cruz per convincerlo ad abbracciare il nuovo stile di vita religiosa. Insieme con lui si recò alla fondazione di Valladolid: “Y como estuvimos algunos días con officiale para recoger la casa, sin clausura, había lugar para informar al padre fray Juan de la Cruz de toda nuestra manera de proceder, para que llevase bien entendidas todas las cosas, así de mortificación como del estilo de hermandad y recreación que tenemos juntas" (De Jesús, 1994: 380-381). Sempre nel Libro de las Fundaciones, testo fondamentale per conoscere i primi passi della Riforma teresiana in Spagna, si racconta come l'anno seguente Juan de la Cruz e il padre Antonio Heredia avessero eretto a Daruelo il primo convento della Riforma maschile. Se in Italia Chiara prende ispirazione e coraggio 
da Francesco d'Assisi, in Spagna il rapporto è rovesciato. E una donna a mostrare il percorso da seguire agli uomini.

In ogni caso la condanna della corruzione della Chiesa - e di ordini monastici troppo indeboliti e fuorviati, come fu il caso di Teresa de Jesús - faceva tutt'uno con l'esortazione alla povertà. Chiara d'Assisi invita all'umiltà e all'abbandono dei beni transitori.

\section{O beata povertà,}

che procura eterne ricchezze

a chi l'ama e l'abbraccia!

O santa povertà,

a quanti l'hanno e la desiderano

Dio promette il regno dei cieli

e ad essi, senza alcun dubbio, sono offerte

la gloria eterna e la vita beata!

\section{O pia povertà,}

che il Signore Gesù Cristo,

che governava e governa il cielo e la terra,

si è degnato di abbracciare più di ogni cosa! (Tonacchera, 1996: 26-27).

Caterina Benincasa, anch'essa avversaria tenace della corruzione ecclesiastica e attiva nel sanare le ferite delle lotte interiori nella sua città, riuscì a convincere papa Gregorio XI a lasciare Avignone per fare ritorno a Roma e si adoperò dunque, da donna ma anche da italiana, come lei scrive, affinché la Chiesa governata e guidata dagli uomini fosse infine salva e riformata. Nei suoi intendimenti, occorreva rendere misericordia al mondo e alla Chiesa con altre armi: “Non con coltello né con guerra né con crudeltà riavarà la bellezza sua; ma con la pace ed umili e continue orazioni, sudori e lagrime, gittate con ansietato desiderio de' servi miei" (S. Caterina da Siena, 1928: 38).

Infine è quella esperienza tutta soggettiva e adogmatica del rapporto con Dio che porterà le mistiche e le contemplative ad essere fondatrici e promotrici di movimenti e conventi: facendosi persino creatrici di pensiero teologico. Vengono in mente Teresa de Jesús e santa Caterina da Siena, ma non può tacersi la potenza anche mentale della voce di Angela da Foligno: quella "maternità spirituale" che fece raccogliere attorno a lei in un autentico cenacolo le anime desiderose di perfezione. A riprova che il fenomeno interessò non solo le donne discendenti da famiglie altolocate che avevano permesso la loro educazione culturale, ma anche quelle del popolo: illetterate, analfabete, persino selvagge nel loro abbraccio della divinità.

\section{RIFERIMENTI BIBLIOGRAFICI}

Alberci, I., Vita della B. Chiara detta della Croce da Montefalco dell'Ordine Eremitano di Sant'Agostino, Roma, appresso Giovanni Battista Robletti, 1610.

Angel da Foligno, Il libro dell'esperienza, a cura di Giovanni Pozzi, Milano, Adelphi, 1992.

----, Il Libro, a cura di Sergio Andreoli, Cinisello Balsamo, Edizioni San Paolo, 2004.

Beer, F., Women and Mystical Experience in the Middle Ages, Woodbridge, The Boydell Press, 1992.

Bell, R. M., Holy Anorexia, Chicago and London, The University of Chicago Press, 1985.

Bevagnati, G., Leggenda della vita e dei miracoli di Santa Margherita da Cortona, Vicenza, Lief, 1978.

----, S. Margherita da Cortona. Vita, colloqui, miracoli, Assisi, Porziuncola Edizioni, 2003.

Borriello, L., Introduzione generale a Opere complete di Teresa d'Avila, Milano, Edizioni Paoline, 1998.

Carpinello, M., Il monachesimo femminile, Milano, Mondadori, 2002.

Caterina Da Siena, S., Libro della Divina Dottrina, a cura di Matilde Fiorilli (II edizione interamente riveduta da Santino Caramella), Bari, Laterza, 1928.

----, Epistolario, a cura di Piero Misciattelli, 2 voll., Firenze, Marzocco, 1939.

Chiara D'Assisi, La Regola, le lettere e il testamento spirituale, a cura di Felice Accrocca, Casale Monferrato, Piemme, 2004.

Claire D'Assise, Écrits, a cura di Marie-France Becker, Jean-François Godet, Thaddée Matura, Paris, Les Éditions du Cerf, 1985 
Criado de Val, M. (a cura di), "Santa Teresa y la literatura mística hispánica", Actas del I Congreso Internacional, Madrid, Edi-6, 1984.

Davy, M.-M., Encyclopédie des mystiques, Paris, Ed. Robert Laffont et Ed. Jupiter, 1972.

De Santi, G., Teresa de Jesús ed altri mistici, Villa Verucchio, Pazzini, 2002.

----,"Santità e poesia: il divino Francesco", in I seminari dell'Umanesimo Latino 2004-2005, Treviso, Fondazione Cassamarca, 2006

----, "Mistiche umbre del Duecento", in Escrituras del Sur. Homenaje a Raffaele Nigro, a cura di Mercedes Arriaga Flórez, Mercedes González de Sande, Ángeles Cruzado Rodríguez e Estela González de Sande, Sevilla, Arcibel Editores, 2009.

Fülöp-Miller, R., Francisco, el santo del amor, Buenos Aires, Espasa-Calpe, 1949.

Fusco, R., Angela, Giuliana e Margherita. Tre mistiche medievali, Milano, Ancora, 2008.

Gennaro, C., "II francescanesimo femminile nel XIII secolo", in Rivista di Storia e Letteratura Religiosa, 25 (1989).

Grundmann, H., Movimenti religiosi nel Medioevo. Ricerche sui nessi storici tra l'eresia, gli ordini mendicanti e il movimento religioso femminile nel XII e XIII secolo e sulle origini storiche della mistica tedesca, Bologna, Il Mulino, 1974.

Herpoel, S., « L'autobiographie au Siècle d'Or. Sainte Thérèse et le 'Libro de Recreaciones' (1585) », in Écrire sur soi en Espagne, Modèles \& Écarts, Études Hispaniques 14, Actes du III Colloque International d'Aix-en-Provence (4-5-6 décembre 1986), Aix-en-Provence, Université de Provence, 1988

Hildegardis Bingensis, Opera minora, ediderunt Peter Dronke, Christopher P. Evans, Hugh Feiss, Beverly Mayne Kinzle, Carolyn A. Muessig, Barbara Newman, Turnhout, Brepols Publishers, 2007.

Jeanne des Anges, Storia della mia possessione, a cura di Angelo Morino, Palermo, Sellerio, 1986.

Leonardi, C. e Menestò, E. (a cura di), "S. Chiara di Montefalco e il suo tempo", Atti del Convegno di studio, Spoleto, 1992.

Llamas Martínez, E., Santa Teresa de Jesús y la Inquisición española, Madrid, C.S.I.C., 1972.

Macola, E., “Nel mistero di un incontro”, in Il Manifesto, XXXIII, n. 130, 30 maggio 2003.

Muraro, L., Il Dio delle donne, Milano, Mondadori, 2003.

Nessi, S. (a cura di), Chiara da Montefalco, badessa del Monastero di S. Croce, Montefalco, Associazione dei Quartieri di Montefalco, 1981

Pásztor, E., Donna e donne. Studi sulla religiosità femminile nel Medio Evo, Roma, Studium, 2000.

Peers, E. A., El misticismo español, Buenos Aires, ESpasa-Calpe, 1947.

Rusconi, R., "L'espansione del francescanesimo femminile nel secolo XIII, in Movimento religioso femminile e francescanesimo nel secolo XIII", Atti del VII Convegno di Studi Francescani, Assisi, Società internazionale di Studi Francescani, 1980
----, "Il movimento religioso femminile in Umbria nei secoli XIII-XIV", Atti del Convegno internazionale di studio nell'ambito delle celebrazioni per l'VIII centenario della nascita di S. Francesco d'Assisi, FirenzePerugia, 1984.

San Francesco, Tutti gli scritti seguiti dai Fioretti, a cura di Henry Furst, Milano, Longanesi, 1972.

S. Juan de la Cruz, Cántico spiritual y poesía completa, Barcelona, Crítica, 2002.

----, Noche obscura, Madrid, Editorial de Espiritualidad, 2003.

Sicari, A., L'itinerario di Santa Teresa d'Avila, Milano, Jaca Book, 1994.

Teresa De Jesús, Obras completas, Madrid, Editorial de Espiritualidad, 1994

Teresa Di Lisieux, Storia di un'anima, Milano, Áncora, 1997.

Théologiens et mystiques au Moyen Âge, édition d'Alain Michel, Paris, Gallimard, 1997.

Tonacchera, R. (a cura di), Mistiche cristiane del Medioevo, Como, Red edizioni, 1996.

Weil, S., La pesanteur et la grâce, Paris, Plon, 1947.

----, Lettre à un religieux, Collection Espoir dirigée par Albert Camus, Paris, Gallimard, 1951.

---, Cahiers, tomes I, II, III, Paris, Plon, 1956

----, Le ravissement de la raison, textes choisis et présentés par Stéphane Barsacq, Paris, Éditions Points, 2009. 